\title{
JUDICIALIZAÇÃO DA SAÚDE: UMA ANÁLISE DA AÇÃO DA JUSTIÇA SOB A GESTÃO DA SAÚdE NO ESTADO DE PERNAMBUCO
}

\author{
F. H. C. de OLIVEIRA ${ }^{1}$, J. E. de LORENA SOBRINHO ${ }^{1}$, U. R. MONTARROYOS ${ }^{5}$, M. G. \\ A. B. das NEVES ${ }^{1}$, P. R. da SILVA ${ }^{1}$, A. P. S. BARBOSA ${ }^{1}$, M. C. S. de LIMA ${ }^{1}$, H. O. \\ BARROS $^{2}$, G. JORGE FILHO ${ }^{3}$, D. M. GUEDES ${ }^{3}$, S. B. de LORENA ${ }^{4}$ \\ ${ }^{1}$ Universidade Estadual de Pernambuco, Faculdade de Ciências Médicas \\ ${ }^{2}$ Universidade Federal de Pernambuco \\ ${ }^{3}$ Secretaria de Estado de Saúde de Pernambuco \\ ${ }^{4}$ Faculdade Pernambucana de Saúde \\ ${ }^{5}$ Universidade Estadual de Pernambuco, Faculdade de Ciências Médicas, Instituto de Ciências \\ Biológicas
}

E-mail para contato: fabiohcavalcanti@upe.br

RESUMO - Este artigo trata da judicialização da saúde no estado de PE, a partir de uma análise da ação desta sob a gestão da saúde. Como de um lado há o direito à saúde, do outro os limites da organização do Estado, vê-se a judicialização da saúde como um fenômeno influenciador da desestruturação da política de medicamentos, mas também de toda a política de gestão financeira do SUS. Para se avaliar o impacto financeiro da ação da justiça sobre a gestão da saúde no estado de PE, usando instrumentos de avaliação econômica, principalmente microcusteio, faz-se necessário inicialmente compreender o perfil da judicialização na SES/PE, objetivo deste trabalho. Usando uma metodologia de trabalho exploratório, retrospectivo, qualitativo e quantitativo, tendo como local para o desenvolvimento do estudo a SES/PE, os resultados obtidos são: medicamentos, que no ano de 2016 identificou-se 63,5\% das ações judiciais impetradas contra a SES/PE; $42 \%$ dessas concentram-se na $1^{a}$ Região de Saúde (RMR Recife); em sua maioria foram patrocinadas por Órgãos Públicos, correspondendo a 54,6\% dos casos, com mais de $95 \%$ de sucesso. Os resultados obtidos mostram sintonia com algumas teses citadas, onde a judicialização da Saúde no Estado de PE é caracterizada pelo crescente número de demandas judiciais por medicamentos, mas potencialmente por impetrantes públicos, neste último caso diferente do já citado em outros trabalhos, reafirmando a necessidade do incentivo e investimentos em pesquisas relacionadas ao tema.

Palavras chave: Economia da saúde, Organização e Administração, Financiamento Governamental.

ABSTRACT - This article deals with the judicialization of health in the state of $P E$, based on an analysis of the health action under the management of health. As on one side there is the right to health, on the other the limits of the organization of the State, we see the judicialization of health as a phenomenon that influences the restructuring of drug policy, but also of the entire financial management policy 
of SUS. In order to evaluate the financial impact of the action of the justice system on health management in the state of PE, using instruments of economic evaluation, mainly micro-accounting, it is necessary to initially understand the profile of the judiciary in SES/PE, objective of this work. Using an exploratory, retrospective, qualitative and quantitative methodology, with the SES/PE as the place of study, the results obtained are: drugs, which in the year 2016, with 63.5\% of the lawsuits filed against SES/PE; 42\% of these are concentrated in the 1st Region of Health (RMR Recife); were mostly sponsored by Public Organs, corresponding to $54.6 \%$ of the cases, with more than $95 \%$ success. The results obtained are in line with some of the aforementioned theses, where the judicialization of Health in the State of PE is characterized by the increasing number of lawsuits for medicines, but potentially by public prosecutors, in the latter case different from what has already been cited in other studies, reaffirming the need incentives and investments in research related to the topic.

Key words: Health Economics, Organization and Administration, Financing, Government

\section{INTRODUÇÃO}

Um impasse se traduz onde: por um lado há o direito à saúde, composto por cuidados relativos à promoção, proteção e recuperação em qualquer nível de atenção (Lei 8.080 de 1990 - SUS, regulamentada pelo Decreto 7.508 de 2011); e de outro os limites próprios da organização do Estado para a prestação das ações e serviços, no concernente à gestão e ao financiamento (CONAS, 2017).

Uma vez essa dualidade apresentada, direito à saúde e limites da organização do Estado, quanto às questões de ordem financeira, em todo o Brasil vê-se um fenômeno "desestruturador" da política de medicamentos, por exemplo, bem como de toda a política de gestão financeira do SUS. Neste aspecto, comprometendo os orçamentos para a aquisição de insumos. De certa forma, tornando-se frequente a reivindicação por parte do cidadão, via sistema judiciário (judicialização na saúde), da aquisição de medicamentos que não constam nas relações de medicamentos essenciais (MESSEDER, 2005, apud VIEIRA, 2007).

Vale ressaltar que a ação da "judicialização na saúde" no Brasil surge no início da década de 1990, com a reivindicação de fornecimento de drogas, principalmente contra o HIV. Com o passar dos anos, o sucesso de ganhos das causas judiciais em torno da saúde, e assim a construção desta jurisprudência, passou a tomar maior proporção e a via de acesso tornouse exemplo para pacientes que sofriam com outras doenças crônicas e raras. Desta forma o poder judiciário começou a se tornar protagonista no processo de garantia à saúde. É um fenômeno que tem sido cada vez mais crescente no âmbito do SUS. No dia a dia dos gestores, há uma percepção de que o usuário do SUS, em sua maioria, tem um caráter imediatista. $\mathrm{Ou}$ seja, quando não conseguem acesso aos serviços e insumos da saúde no tempo desejado, acabam, na maioria das vezes, impetrando em juízo e requerendo o que a Constituição Federal de 1988 lhes assegura como direito fundamental (SILVA JÚNIOR, 2016).

Em Pernambuco, identificam-se movimentos para lidar com a problemática da judicialização, com destaque para a estruturação do Núcleo de Assessoria Técnica em Saúde (NATS) firmado através de convênio de cooperação técnica (Convênio no 048/2011) entre a Secretaria Estadual de Saúde de Pernambuco (SES-PE) e o Tribunal de Justiça de Pernambuco (TJPE) e a estruturação do Núcleo de Ações Judiciais (NAJ), em maio de 2014, na SES-PE.

Para se avaliar o impacto financeiro da ação da justiça sobre a gestão da saúde no estado 
de Pernambuco, usando instrumentos de avaliação econômica, principalmente microcusteio, faz-se necessário inicialmente compreender o perfil da judicialização na Secretaria Estadual de Saúde de Pernambuco, objetivo deste trabalho.

A partir deste objetivo traçado, por meio de metodologia de trabalho exploratório, retrospectivo, qualitativo e quantitativo, como local para o desenvolvimento do estudo a $\mathrm{SES} / \mathrm{PE}$, os resultados obtidos são curiosos e justificam por si só o trabalho e o estimulo a pesquisa sobre o tema.

Observa-se que os medicamentos, como citado em 2016, possuem 63,5\% das ações judiciais impetradas contra a SES/PE, no entanto $42 \%$ desses concentram-se na $1^{\text {a }}$ Região de Saúde da Região Metropolitana do Recife - RMR, fato este por muitos outros autores reforçado, ou seja, medicamentos são os potencialmente produtos de judicialização. No entanto um dado divergiu da literatura posta, os Órgãos Públicos, 54,6\% dos casos, foram os que mais impetraram ações contra a SES/PE, com mais de $95 \%$ de sucesso. A participação de impetrantes públicos denota algo a se investigar, pois há relatos em trabalhos que apontam a judicialização como um processo das elites brasileiras (MEDEIROS et al, 2011).

\section{MATERIAIS E MÉTODOS}

Sendo um trabalho exploratório, retrospectivo, qualitativo e quantitativo, define-se como local para o desenvolvimento do estudo a SES/PE.

O levantamento de dados foi realizado no NAJ/SES-PE. As informações foram obtidas através do banco de dados "Ações judiciais 2016" disponibilizado pelo Núcleo, tendo como recorte temporal o ano de 2016, contendo 2.560 processos.

As variáveis de interesse escolhidas foram: o produto; a unidade de atendimento (se SUS, rede privada e filantrópico); condição patológica segundo a CID-10; municípios da comarca; município de residência; sexo; grupo do produto (se medicamento, UTI, alimento, insumo, material médico-hospitalar, cirurgia, equipamento, homecare, exame, consulta, Órteses, Próteses e Materiais Especiais, internamento e outros) e condutor da ação (se Defensoria Pública Da União - DPU, Ministério Público Da União- MPU, Associação De Defesa Dos Usuários De Seguros, Planos E Sistemas De Saúde - ADUSEPS, Defensoria Pública Do Estado De Pernambuco - DPPE, Ministério Público De Pernambuco- MPPE e particular).

Os dados foram tabulados em Excel, onde foram calculadas as frequências simples e os percentuais de todas as variáveis. No segundo momento, foi realizado o cruzamento das seguintes variáveis: produto versus condição patológica segundo a CID-10; produto versus a unidade de atendimento; condição patológica segundo a CID-10 versus Região de Saúde de residência; condutor da ação versus produto; condutor da ação versus Região de Saúde de residência e produto versus Região de Saúde de residência.

\section{RESULTADOS E DISCUSSÃO}

Dentre as demandas judiciais relacionadas à saúde no Estado de Pernambuco, destacamse os medicamentos, que no ano de 2016 com a 63,5\% das ações judiciais impetradas contra a SES/PE (Tabela 1). Este cenário corrobora com os estudos encontrados na literatura, que afirmam que os medicamentos para uso individual são os principais objetos de judicialização da saúde (MESSEDER AM et al., 2005). PEPE e SCHRAMM (2010) identificaram que houve um aumento considerável no número de processos judiciais individuais e coletivos principalmente como via alternativa de aquisição de medicamentos no Sistema Único de Saúde - SUS. Diniz et al (2014) complementam ainda com um estudo realizado no Distrito 
Federal no ano de 2013, onde identificaram que entres os anos 2005 a 2010 houve um aumento permanente da judicialização na saúde.

A demanda por medicamentos no ano de 2016 em PE inclui 832 fármacos distintos, sendo os 5 medicamentos mais requisitados: Cinacalcete, Somatropina, Abiraterona, Insulina Glargina e Leuprorrelina. O Cinacalcete (Mimpara ${ }^{\circledR}$ ) é o medicamento mais judicializado no Estado de Pernambuco, correspondendo a 4,5\% das demandas de medicamentos. $\mathrm{O}$ fármaco é indicado para o tratamento de pacientes com Hiperparatireoidismo secundário (HPTS) à doença crônica renal (DRC), que são submetidos à diálise com paratormônio (PTH) superior a $800 \mathrm{pg} / \mathrm{mL}$. Este foi incorporado através da Portaria $\mathrm{N}^{\circ} 48$ da Secretaria de Ciência, Tecnologia e Insumos Estratégicos do Ministério da Saúde em 29 de setembro de 2015, com prazo de 180 dias para incorporação. Contudo, em novembro de 2016 o Protocolo Clínico e Diretrizes Terapêuticas Específico foi aberto para uma segunda consulta pública e até o momento da redação deste artigo não começou a ser distribuído, podendo explicar o crescente número de demandas judiciais para aquisição do Cinacalcete (CONITEC, 2015).

Tabela 1 - Demandas judiciais por Categoria. Pernambuco, 2016. Elaborada pelos autores a partir de Banco de dados com as ações judiciais do ano de 2016, do Núcleo de Ações Judiciais - NAJ da Secretaria Estadual de Saúde de Pernambuco- SES/PE.

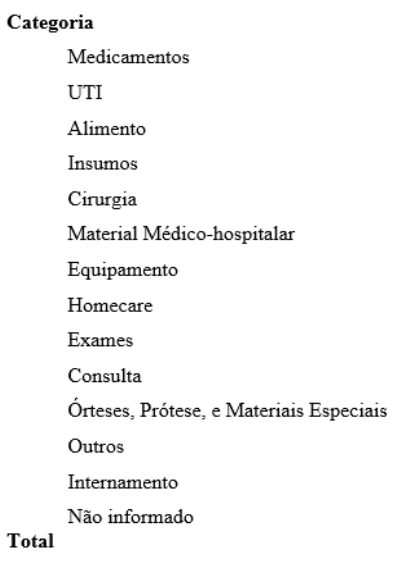

$\begin{array}{ll}\mathbf{N} & \text { \% } \\ 1626 & 63,5 \\ 207 & 8,1 \\ 131 & 5,1 \\ 119 & 4,6 \\ 94 & 3,6 \\ 86 & 3,4 \\ 73 & 2,8 \\ 66 & 2,6 \\ 55 & 2,1 \\ 36 & 1,4 \\ 25 & 1,0 \\ 23 & 1,0 \\ 18 & 0,7 \\ 1 & 0,04 \\ \mathbf{2 5 6 0} & \mathbf{1 0 0}\end{array}$

Quando distribuídas as demandas nas 12 regiões de saúde, observa-se que $42 \%$ dessas concentram-se na $1^{\text {a }}$ Região de Saúde, que corresponde em sua maioria à Região Metropolitana do Recife. Esse fato poder ser justificado pelo grande aglomerado de pessoas na região como também pela concentração de unidades de saúde de média e alta complexidade. Além disso, foi observado que as unidades de origem das ações em sua maioria do SUS e Filantrópicos, representando $39,3 \%$ e $31,5 \%$ respectivamente. A rede privada representa $19,3 \%$.

No Estado de Pernambuco, durante o período de análise, foi observado que as demandas judiciais em sua maioria foram patrocinadas por Órgãos Públicos, correspondendo a 54,6\% (Gráfico 1)dos casos, sendo a DPPE o órgão com maior representatividade no número de casos, com $36,8 \%$. 
Gráfico 1 - Ações judiciais por patrocinador. Pernambuco, 2016. Fonte: Banco de dados com as ações judiciais do ano de 2016, do Núcleo de Ações Judiciais - NAJ da Secretaria Estadual de Saúde de Pernambuco- SES/PE.

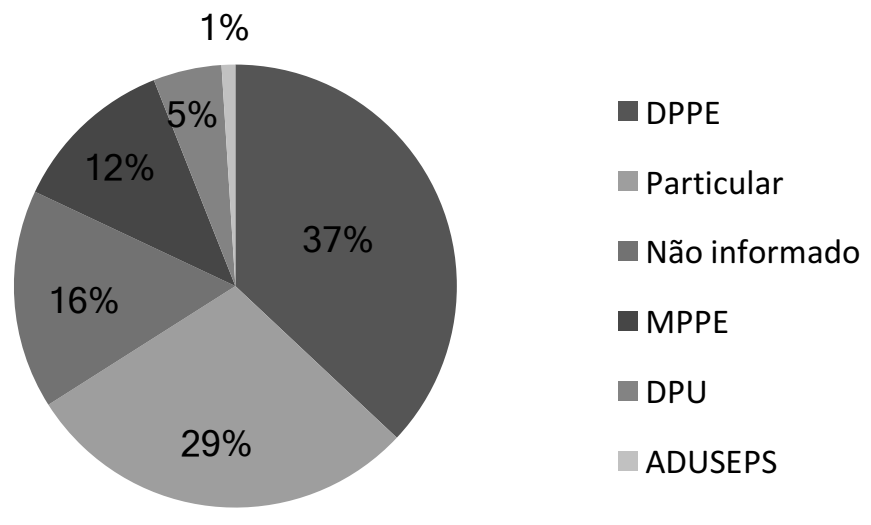

Das 2.560 ações judiciais impetradas contra a Secretaria de Saúde de Pernambuco no ano de 2016, 2.438 foram deferidas, correspondendo a 95,2\% e apenas 4,5\% (115) tiveram o pedido negado.

\section{CONCLUSÃO}

Os resultados obtidos mostram que assim como em algumas teses encontradas na literatura, a judicialização da Saúde no Estado de Pernambuco é caracterizada pelo crescente número de demandas judiciais por medicamentos, configurando-se como via de acesso alternativa para aquisição destes no SUS, uma vez que os fármacos mais requisitados estão incorporados na lista de Assistência Farmacêutica do MS.

As demandas judiciais do Estado concentram-se na $1^{\mathrm{a}}$ Região de Saúde, ou seja, região metropolitana do Recife, vindas predominantemente de unidades de saúde do SUS e patrocinadas comumente por órgãos públicos, não corroborando com literaturas que afirmam que a judicialização se caracteriza como um movimento da elite brasileira.

O sucesso das ações foram superiores a $95 \%$.

Além disso, o estudo reafirma a necessidade do incentivo e investimentos em pesquisas relacionadas ao tema, tendo em vista a complexidade e relevância do assunto para a saúde pública brasileira.

\section{REFERENCIAS}

BRASIL. Constituição Federal. Brasília, 1988._. Decreto 7.508. Brasília, 2011.

CONSELHO NACIONAL DE SECRETÁRIOS DE SAÚDE - CONAS. Institucional. Brasil. Disponível em: http://www.conass.org.br/. Acesso em: 06 nov. 2017. 
DINIZ, D. et al. A judicialização da saúde no Distrito Federal, Brasil. Ciência \& Saúde Coletiva 19.2 (2014): 591-598.

MESSEDER, A. M. et al. Mandados judiciais como ferramenta para garantia do acesso a medicamentos no setor público: a experiência do Estado do Rio de Janeiro, Brasil. Cadernos de Saúde Pública, Rio de Janeiro, v.21, n.5, p525-534, mar./abr. 2005.

PEPE, V. L. E.; SCHRAMM, F. R. Judicialização da saúde, acesso à. Physis Revista de Saúde Coletiva 20.1 (2010): 77-100.

SECRETARIA ESTADUAL DE SAÚDE DE PERNAMBUCO - SES/PE. A secretaria. Pernambuco. Disponível em: http://portal.saude.pe.gov.br/secretaria. Acesso em: 17 ago. 2017.

VIEIRA, F.S.; ZUCCHI, P. Distorções causadas pelas ações judiciais à política de medicamentos no Brasil. Rev Saúde Pública 2007;41(2):214-22.

\section{AGRADECIMENTOS}

Às autoras Mirlene das NEVES, Paula da SILVA, que concluíram seus Trabalhos de Conclusão de Curso em Saúde Coletiva na UPE/FCM, optando pelo tema de alta relevância, aos seus orientadores José de LORENA SOBRINHO e Maria Cristina Sete de LIMA, o superintendente do NAJ Geraldo Jorge Filho e ao farmacêutico Diego Medeiros Guedes e todos que contribuíram para a realização deste trabalho. Além das Instituições sede de pesquisa, UPE/FCM e SES/PE. 